\title{
Migrating neuroblasts in the adult human brain: a stream reduced to a trickle
}

\author{
Miriam E van Strien ${ }^{1}$, Simone A van den Berge ${ }^{1}$, Elly M Hol ${ }^{1}$ \\ ${ }^{I}$ Department of Astrocyte Biology and Neurodegeneration, Netherlands Institute for Neuroscience, an institute of the Royal Nether- \\ lands Academy of Arts and Sciences, Meibergdreef 47, 1105 BA, Amsterdam, The Netherlands \\ Cell Research (2011) 21:1523-1525. doi:10.1038/cr.2011.101; published online 21 June 2011
}

It has long been thought that neurogenesis (birth of neurons) in the mammalian brain only occurs while the central nervous system is still developing. Although the first indications to the contrary already appeared in the 1960s, it took more than 30 years for the neuroscience community to accept that the mammalian adult brain also generates new neurons. Today it is completely accepted that neurogenesis occurs in two mammalian adult brain areas, the subventricular zone (SVZ) near the lateral ventricles and the subgranular zone in the hippocampus. The SVZ is the largest neurogenic niche where neural stem cells reside. These stem cells are B cells, a subtype of astrocytes, which generate neuroblasts via a transitamplifying neural progenitor. In rodents and primates, these neuroblasts migrate in chains into the olfactory bulb, where they differentiate into interneurons. This migratory path is called the rostral migratory stream (RMS) [1].

A breakthrough that gave the adult neurogenesis field an enormous boost was the discovery of neural stem cells in the adult human brain. In humans, the main neurogenic niches are also the SVZ $[2,3]$ and the hippocampus [3]. The neural stem cells in the human

Correspondence: Elly M Hol

E-mail: e.hol@nin.knaw.nl
SVZ reside in a ribbon of neurogenic astrocytes [2], initially identified by the expression of the astrocyte-specific protein "glial fibrillary acidic protein" (GFAP) and the co-expression of cell proliferation markers (Ki67 and PCNA) $[2,4]$. Further evidence of the astrocytic nature of neural stem cells came from the incorporation of the synthetic analogue of thymidine, BrdU, in the DNA of proliferating GFAP-positive cells in organotypic slice cultures of the adult human SVZ, and from the ability of these SVZ astrocytes to generate clonal neurospheres [2]. The primate (including human) SVZ neurogenic niche appears to have many similarities with the rodent SVZ niche, although there are also distinct differences, such as their anatomical organization, in the sense that the human neurogenic astrocytes are separated from the ependymal layer by a hypocellular gap [2,5]. Such a hypocellular gap has also been observed in some primates [6]. Another major difference is the structure and composition of the RMS. An RMS has been identified in rodents and primates $[1,6]$, but the debate on whether a true RMS is present in the human brain is still ongoing. A recent paper by Wang et al. [7] provides important data relevant to this discussion.

In the adult rodent brain, the RMS is a robust stream of neuroblasts that are born in the SVZ neurogenic niche, migrate in chains along the RMS into the olfactory bulb and replace olfactory bulb interneurons [1]. Determining whether a stream of migrating cells is present is highly dependent on the availability of specific markers of migrating cells. In the human brain, proliferating cells are mainly identified by endogenous cell proliferation markers, such as PCNA, pHH3, Mcm2 and Ki67, neuroblasts by PSA-NCAM and doublecortin (DCX), and differentiating neurons by NeuN and $\beta$-III tubulin/TuJ1. The initial paper of Sanai et al. [2], in which the neurogenic astrocytes in the SVZ are described as the stem cells of the human brain, explicitly states that the lack of cells expressing the migrating neuroblast marker PSA-NCAM and the immature young neuron marker TuJ1 indicated that there was no evidence for the presence of an RMS. Furthermore, the olfactory peduncle, a structure that any and all migrating neuroblasts must pass, was devoid of chain migrating TuJ1-positive cells. However, these authors also describe that they seldom observed an elongated cell that was TuJ1 positive, but that such a young neuron did not migrate in chains as it did in the rodent and primate brain. In a later paper they showed the presence of a few PSA-NCAM-positive cells, implying that some migrating cells are present, but these were not positive for DCX [5]. They [2, 5] concluded that 
only a remnant of the stream is present in the adult human brain based on the absence of chain migrating neuroblasts, and the presence of a few cells that express migration markers and the presence of ependymal cavities along an RMS-like path. A year later, Curtis et al. [8] stated that the human brain does have an RMS. This meant the start of a debate on whether the stream is present or not [9] and what its structure is. The conclusion of Curtis et al. was based on the presence in the brain of a stream-like structure composed of PCNA-positive cells. They also showed that the RMS in the human brain is anatomically different from that in the rodent and primate brain. The stream starts at the frontal part of the lateral ventricle and first takes a caudal path. According to Curtis et al., the descending limb is organized near a ventricular extension, after which it enters the olfactory tract. This ventricular extension is called the
SVZ-olfactory-trigone connection by Sanai et al. [2,9] and is the region that housed the olfactory ventricle in early human development [9]. The recent paper by Wang and colleagues [7] reports, for the first time, the presence of DCX-expressing cells in the human adult RMS, implying that these cells are indeed migrating. The DCX-positive cells often appeared in pairs, indicating that the cells divided symmetrically and most likely were the offspring of neuroblasts. Migrating cells were only identified in the ventral SVZ and along the RMS, but not in the olfactory bulb. This is not very surprising, as it is known from rodent studies that only a small percentage of migrating cells will integrate into the olfactory bulb [1]. The data of Wang et al. confirms that the human RMS exists, but that only a few migrating neuroblasts follow this stream. This is in agreement with the earlier findings of Sanai et al. and
Quinones-Hinojosa et al. The hollow cerebrospinal fluid-filled ventricle in the olfactory bulb observed by Curtis et $a l$. was not confirmed by either Sanai et $a l$. or Wang et al. Evidence thus appears to be accumulating for the human adult brain to contain a RMS, albeit one with only a few migrating cells.

The paper by Wang et al. also offers evidence for the presence of a stream of DCX-positive cells in a foetal RMS (gestational week 22-24). This confirms an extensive analysis of the cytoarchitecture of the human foetal brain (gestational week 23-24) by Guerrero-Cázares et al. [10], which postulates that the RMS appears to be a real stream, complete with chain migrating neurons in the developing brain. In the adult brain, the stream becomes a trickle (see Figure 1). However, it must be noted that mainly brains over the age of 35 were analyzed and that until now the presence of an RMS has
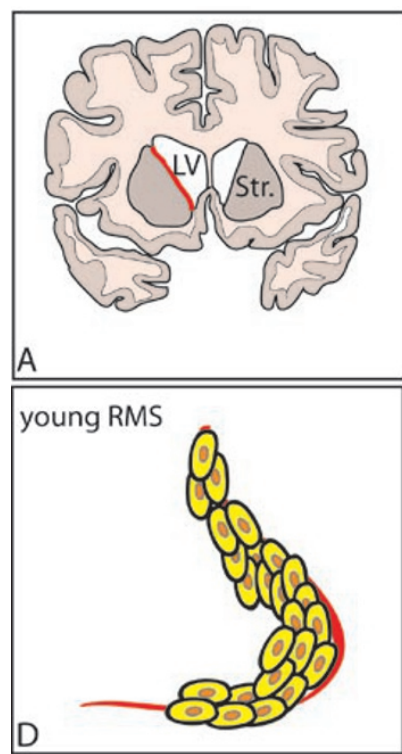

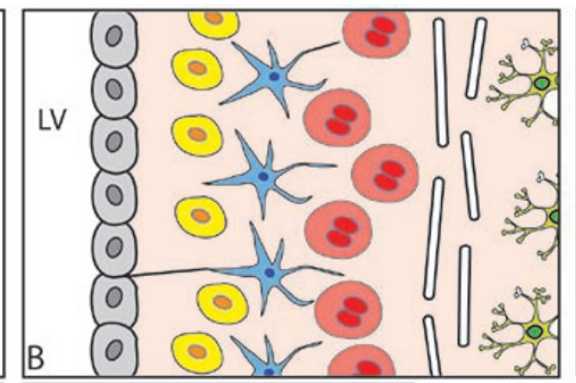

old RMS

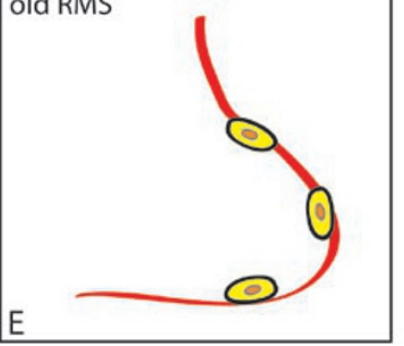

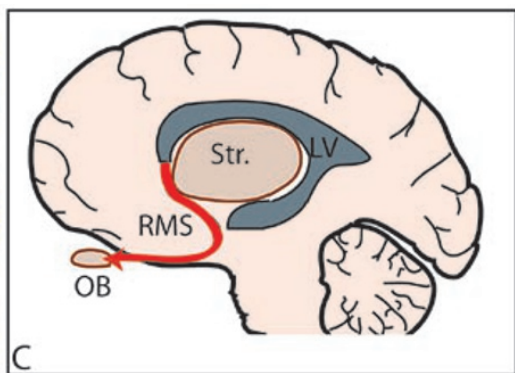

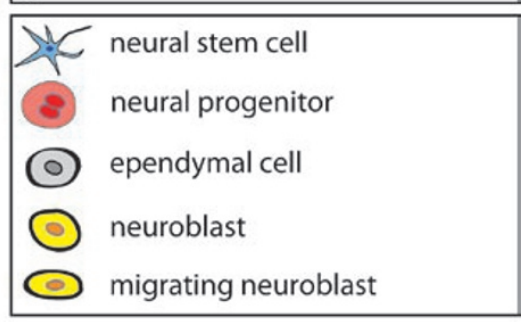

Figure 1 The adult human neurogenic system. (A) Location of the subventricular zone (SVZ; red line) in a coronal section of the human brain, where the SVZ lies between the lateral ventricle (LV) and the striatum (Str.). (B) Cellular composition of the SVZ. Ependymal cells (grey) separate the lateral ventricle from the SVZ, which consists of four main cell types: niche astrocytes (not shown), neurogenic astrocytes/neural stem cells (blue), some of them touching the ependymal cells layer, amplifying neural progenitor cells (red) and neuroblasts (yellow). The SVZ is separated from the underlying striatum (on the right side of the image) by a myelin layer (white bars). (C) Sagittal view of the RMS (RMS; red arrow), by which the neuroblasts migrate to the olfactory bulb (OB). (D) In the young RMS, chain migration of migrating neuroblasts takes place whereas (E) in the old RMS, only a few migrating neuroblasts are present that are positive for the neuroblast/young neuronal markers DCX, PSA-NCAM and $\beta$-III tubulin. 
only been observed in just a few adult human brains. It is currently not known at which precise time point the number of migrating neuroblasts in the human brain begins to decline. A clear agerelated reduction in neurogenesis in the hippocampal niche has been reported for rodents and a similar age-related decline in DCX-positive cells in the human hippocampus was shown [11]. The effect of aging on neurogenesis in the SVZ has not been extensively studied. In mice it was shown that cell proliferation is reduced in the SVZ in middle age (12 months of age) compared to young adults ( 2 months of age) and that this is reflected in a reduction of neuroblasts in the olfactory bulb, implying that fewer neuroblasts travel along the RMS in the older mice [12]. Such a decline is also expected for the human SVZ, but so far no evidence of this has been published. We have observed neural stem cells in the aged human SVZ, but due to the enormous individual variation we did not find a significant correlation between the number of stem cells and age [4].

Determining whether migrating neuroblasts are indeed present in an RMS in the adult human brain is important, as this would prove that the neural stem cells in the SVZ in the adult brain are still capable of generating neuroblasts able to migrate and potentially differentiate and integrate into a neuronal network. The SVZ is closely situated near the lateral ventricles, and therefore the neural stem cells are accessible for manipulation by intracerebroventricular delivery of growth factors. The discovery of the presence of intact neural stem cells in the adult SVZ able to spawn neuroblasts thus opens new avenues for the development of novel strategies to stimulate these cells to replace damaged neurons and glia. The brain's potential for self-repair appears from studies showing that the proliferation of neural stem cells in the SVZ increases after stroke $[13,14]$ and in Huntington's disease, a neurodegenerative disorder [15]. However, this endogenous response clearly does not suffice as far as preventing neurodegeneration and brain damage are concerned.

\section{Acknowledgments}

We would like to acknowledge the financial support of NWO-ALW-Vici (865.09.003 to EMH), the "Internationaal Parkinson Fonds" and the "DorpmansWigmans Stichting". We also thank Wilma Verweij (Netherlands Institute for Neuroscience, The Netherlands) for critically reading the manuscript.

\section{References}

1 Whitman MC, Greer CA. Adult neurogenesis and the olfactory system. Prog Neurobiol 2009; 89:162-175.

2 Sanai N, Tramontin AD, QuinonesHinojosa A, et al. Unique astrocyte ribbon in adult human brain contains neural stem cells but lacks chain migration. Nature 2004; 427:740-744.

3 Eriksson PS, Perfilieva E, BjorkEriksson T, et al. Neurogenesis in the adult human hippocampus. Nat Med 1998; 4:1313-1317.

4 van den Berge SA, Middeldorp J, Zhang $\mathrm{CE}$, et al. Longterm quiescent cells in the aged human subventricular neurogenic system specifically express GFAP-delta. Aging Cell 2010; 9:313326.

5 Quinones-Hinojosa A, Sanai N, Soriano-Navarro $\mathrm{M}$, et al. Cellular composition and cytoarchitecture of the adult human subventricular zone: a niche of neural stem cells. J Comp Neurol 2006; 494:415-434.

6 Sawamoto K, Hirota Y, Alfaro-Cervello $\mathrm{C}$, et al. Cellular composition and organization of the subventricular zone and rostral migratory stream in the adult and neonatal common marmoset brain. J Comp Neurol 2011; 519:690713.

7 Wang C, Liu F, Liu YY, et al. Identification and characterization of neuroblasts in the subventricular zone and rostral migratory stream of the adult human brain. Cell Res 2011; 21:1534-1550.

8 Curtis MA, Kam M, Nannmark U, et al. Human neuroblasts migrate to the olfactory bulb via a lateral ventricular extension. Science 2007; 315:12431249.

9 Sanai N, Berger MS, Garcia-Verdugo JM, Alvarez-Buylla A. Comment on "Human neuroblasts migrate to the olfactory bulb via a lateral ventricular extension". Science 2007; 318:393.

10 Guerrero-Cazares H, Gonzalez-Perez $\mathrm{O}$, Soriano-Navarro $\mathrm{M}$, et al. Cytoarchitecture of the lateral ganglionic eminence and rostral extension of the lateral ventricle in the human fetal brain. J Comp Neurol 2011; 519:11651180.

11 Knoth R, Singec I, Ditter M, et al. Murine features of neurogenesis in the human hippocampus across the lifespan from 0 to 100 years. PLoS One 2010; 5:e8809.

12 Bouab M, Paliouras GN, Aumont A, Forest-Berard K, Fernandes KJ. Aging of the subventricular zone neural stem cell niche: evidence for quiescenceassociated changes between early and mid-adulthood. Neuroscience 2011; 173:135-149.

13 Jin $\mathrm{K}$, Wang X, Xie L, et al. Evidence for stroke-induced neurogenesis in the human brain. Proc Natl Acad Sci USA 2006; 103:13198-13202.

14 Marti-Fabregas J, Romaguera-Ros M, Gomez-Pinedo U, et al. Proliferation in the human ipsilateral subventricular zone after ischemic stroke. Neurology 2010; 74:357-765.

15 Curtis MA, Penney EB, Pearson AG, et al. Increased cell proliferation and neurogenesis in the adult human Huntington's disease brain. Proc Natl Acad Sci USA 2003; 100:9023-9027. 